\title{
Manejo cirúrgico do paciente submetido à terapia anticoagulante oral
}

\author{
Surgical management of patients undergoing oral anticoagulant therapy \\ Manejo quirúrgico del paciente sometido a la terapia anticoagulante oral \\ Thaís Evellyn da Silva ${ }^{1}$, Emanuele Castro de Araújo², Marcela Paschoal Rocha ${ }^{3}$, Leandro Moraes \\ Coelho de Oliveira
}

Como citar esse artigo. da Silva, TE; de Araújo, EC; Rocha, MP; de Oliveira, LMC. Manejo cirúrgico do paciente submetido à terapia anticoagulante oral Revista Pró-UniverSUS. 2019 Jan./Jun.; 10 (1): 145-149.

\section{Resumo}

Introdução: bem se sabe que quandoumpaciente está submetidoàterapia anticoagulante, seus níveis de coagulaçãoficamalterados, aumentando o risco de intercorrências transoperatórias. Dessa forma, tem-se levantado muitos questionamentos, tanto na área médica como na odontológica, quanto à melhor conduta a se seguir para a realização de um tratamento cirúrgico-odontológico seguro em pacientes anticoagulados. Com o objetivo de realizar uma revisão de literatura abordando as peculiaridades inerentes ao atendimento adequado e com segurança desses pacientes, elucidando o método de diagnóstico, os exames pré-operatórios a serem solicitados, bem como os riscos existentes na manutenção ou interrupção da terapia anticoagulante a qual os pacientes estão sendo submetidos. Materiais e Métodos: Foi realizada uma busca na base de dados Scientific Electronic Library Online (SCIELO), PubMed e Google acadêmico com os descritores: "surgery oral", "anticoagulants" e "toothextraction", no mês de Setembro de 2018, onde foram levantados 25 artigos e foram selecionados 18 artigos de acordo com os critérios de inclusão. Conclusão: Foi observado que a manutenção da terapêutica anticoagulante oral vem sendo preconizada, considerando o valor da Razão normalizada internacional (RNI) dentro do adequado como de sumaimportância, assim como o uso de alguma medida hemostática local, bem como as devidas instruções para o período de pós-operatório.

Palavras-chave: Cirurgia bucal, Anticoagulantes, Extração dentária.

\begin{abstract}
Introduction: it is well known that when a patient is submitted to anticoagulant therapy, their coagulation levels remain altered, increasing the risk of transoperative intercurrences events. In this way, many questions have been raised, in a medical area as well as in dentistry, how much better is it to go to a safe clinical-dental treatment in anticoagulated patients. With the objective of conducting a literature review addressing the peculiarities inherent to the adequate and safe care of these patients, elucidating the diagnostic method, the preoperative examinations to be requested, as well as the existing risks In the maintenance or interruption of the anticoagulant therapy to which the patients are being submitted. Materials and Methods: A search was conducted in the Scientific Electronic Library Online (SCIELO), PubMed and Google academic database with the descriptors: "Oral Surgery", "anticoagulants" and "tooth extraction", in September 2018, where 25 Articles and 18 articles were selected according to the inclusion criteria. Conclusion: It has been observed that maintenance of oral anticoagulant therapy has been recommended, considering the value of International Normalized Ratio (INR) within the appropriate as extremely important, as well as the use of some local hemostatic measure, as well as the appropriate instructions for the period of postoperative.

Keywords: Oral Surgery, Anticoagulants, Tooth extraction.
\end{abstract}

\section{Resumen}

Introducción: bien se sabe que cuando un paciente está sometido a la terapia anticoagulante, sus niveles de coagulación quedan alterados, aumentando el riesgo de intercurrencias transoperatorias. De esta forma, se han levantado muchos cuestionamientos, tanto en el área médica como en la odontológica, en cuanto a la mejor conducta a seguir para la realización de un tratamiento quirúrgico-odontológico seguro en pacientes anticoagulados. Con el objetivo de llevar a cabo una revisión bibliográfica que aborde las peculiaridades inherentes a la atención adecuada y segura de estos pacientes, elucidatando el método de diagnóstico, los exámenes preoperatorios a solicitar, así como los riesgos existentes En el mantenimiento o interrupción de la terapia anticoagulante a la que se someten los pacientes. Materiales y métodos: una búsqueda se llevó A cabo en la biblioteca electrónica científica en línea (SCIELO), PubMed y la base de datos académica de Google con los descriptores: "cirugía oral", "anticoagulantes" y "extracción de dientes", en septiembre 2018, donde 25 Se seleccionaron artículos y 18 artículos de acuerdo con los criterios de inclusión. Conclusión: Se observó que el mantenimiento de la terapéutica anticoagulante oral viene siendo preconizada, considerando el valor de la Raza normalizada internacional (RNI) dentro del adecuado como de sumaimportancia, así como el uso de alguna medida hemostática local, así como las debidas instrucciones para el período de post- -operatively.

Palabras clave: Cirugía bucal, Anticoagulantes, Extraccion dentária.

Afiliação dos autores:

1Cirurgiã-Dentista graduada pela Universidade de Vassouras. RJ, Brasil. Email: thais-evellyn@hotmail.com ORCID: https://orcid.org/0000-0002-0547-6222

2Cirurgiã-Dentista graduada pela Universidade de Vassouras. RJ, Brasil. Email: emanuelecaraujo96@gmail.com ORCID: https://orcid.org/0000-0002-4524-1102

3Cirurgiã-Dentista graduada pela Universidade de Vassouras. RJ, Brasil. Email: marcela.procha@hotmail.com ORCID: https://orcid.org/0000-0002-0519-7979

4Mestrando em Saúde Coletiva pela São Leopoldo Mandic, Campinas. SP Brasil. Email: leandromcoliveira@hotmail.com ORCID: https://orcid.org/0000-0003-0944-8445

* Email de correspondencia: thais-evellyn@hotmail.com 


\section{Introdução}

Com o aumento da expectativa de vida e os avanços nas áreas da saúde, é cada vez mais frequente $o$ atendimento odontológico de pacientes com problemas sistêmicos, dentre eles os que estão submetidos à terapia anticoagulante. Por essa razão, o cirurgião-dentista deve estar preparado para o atendimento dessa população, bem como deve estar atento a uma detalhada anamnese, exame clínico e físico. ${ }^{1}$

O exame físico geral deve ser realizado meticulosamente, procurando identificar sinais de problemas hematológicos, procurando por lesões de púrpura, contusões grandes ou profundas, hematomas evidentes, inchaços nas articulações, sangramento nasal e gengival espontâneos. O diagnóstico precoce dessas manifestações clínicas propicia um consequente tratamento mais eficaz. ${ }^{1,2}$

Em relação à administração da terapia anticoagulante, esta pode ser feita por via endovenosa, oral e subcutânea como tratamento profilático ${ }^{3}$. Como causas motivadoras para sua indicação, pode-se citar a prevenção de trombose venosa profunda, embolia pulmonar, trombose e embolização em pacientes com fibrilação atrial e trombose de válvulas cardíacas. É aplicada também em pacientes com prótese cardíaca, para síndromes coronarianas agudas, para pacientes que fazem uso de stents coronarianas, pós-acidentes vasculares isquêmicos cerebrais e cardíacos. ${ }^{4}$

Para que não haja eventuais intercorrências, deve-se realizar um planeamento em três etapas: préoperatório, intra-operatório e pós-operatório. Uma vez que a cirurgia oral em pacientes que fazem uso da medicação, ainda hoje, é um desafio para os clínicos, devido à susceptibilidade hemorrágica apresentada por eles. ${ }^{5,6}$

A opção de manter, suspender, atrasar ou alterar a terapia anticoagulante no pré-cirúrgico dependerá do risco hemorrágico individual e dos procedimentos a serem realizados, assim como do tipo de terapia anticoagulante administrada. Este tipo de decisão deve ser, sempre que possível, uma decisão conjunta entre o médico e o cirurgião-dentista. ${ }^{6}$

A segurança do tratamento anticoagulante oral depende fundamentalmente de controle freqüente e cuidadoso, pois somente dessa forma poderão ser evitadas as complicações tanto trombóticas quanto hemorrágicas, permitindo que o paciente continue se beneficiando dessa modalidade de tratamento. ${ }^{2}$

Considerando que pacientes usuários de anticoagulantes orais se tornaram rotina constante no consultório odontológico, o objetivo deste trabalho foi buscar na literatura a melhor conduta de atendimento a estes pacientes, através da identificação das principais alterações fisiológicas provocadas pelo uso destes anticoagulantes, permitindo assim um correto manejo clinico, evitando, portanto possíveis intercorrências.

\section{Metodologia}

Este estudo trata-se de uma Revisão Integrativa (RI) da literatura. A RI é um método que tem como finalidade reunir e resumir o conhecimento científico já produzido sobre o tema investigado, permitindo buscar, avaliar e sintetizar as evidências disponíveis para contribuir com o desenvolvimento do conhecimento na temática.

EstaRI foi constituída por seis etapas: identificação do tema e seleção da hipótese ou questão norteadora; estabelecimento de critérios de inclusão e exclusão de estudos/amostragem ou busca na literatura; definição das informações a serem extraídas ou categorização dos estudos; avaliação dos estudos incluídos na RI; interpretação dos resultados; apresentação da revisão. Após a elaboração da questão norteadora, foram eleitos como critérios de inclusão: artigos na íntegra disponíveis on-line que abordassem a temática do estudo; apresentação de resumo para primeira apreciação; procedência brasileira ou estrangeira, nos idiomas português, inglês e espanhol.

Como critério de exclusão adotou-se: estudos que citavam hemofilia crônica hereditária. A partir da leitura e da análise dos artigos, com basenas evidências científicas, optou-se por construir um protocolo de orientações préoperatórias, solicitação de exames, parâmetros de Razão Normalizada Internacional (International Normalized Ratio - INR), suspensão ou manutenção da medicação; orientações intraoperatórias, para determinar extensão e complexidade do procedimento, assim como o uso de técnicas hemostáticas locais; orientações e cuidados pós-operatórias. A busca foi realizada nas bases de dados on-line: Scientific Electronic Library Online (SCIELO) e Pubmed. A coleta dos dados ocorreu no mês de Setembro de 2018.

\section{Resultados}

A hemostasia é um processo complexo que envolve diversos eventos fisiológicos. Quando um vaso sanguíneo é lesado, ocorre uma vasoconstrição acentuada. As plaquetas aderem à superfície danificada e se agregam para formar um tampão hemostático temporário, que é a primeira fase da coagulação ou hemostasia primária. Em seguida, ocorre a segunda fase da coagulação ou hemostasia secundária, que acontece através de duas vias separadas: a via intrínseca e a via extrínseca, que fazem parte da cascata da coagulação. ${ }^{7}$

Em condições fisiológicas, essas reações resultam numa produção equilibrada de quantidades apropriadas de trombina e do coágulo de fibrina, em resposta 
adequada e proporcional ao dano vascular existente. $\mathrm{O}$ início do processo de coagulação depende da exposição do sangue a componentes que, normalmente, não estão presentes no interior dos vasos, mas que surgem devido a danos vasculares ou alterações bioquímicas, como por exemplo, a libertação de citocinas. ${ }^{8}$

As medidas locais de hemostasia devem fazer parte do arsenal no atendimento ao paciente anticoagulado. É de fundamental importância que qualquer cirurgião-dentista esteja familiarizado com técnicas e métodos de hemostasia local. Além disso, há um destaque na necessidade do emprego de técnicas cirúrgicas menos traumáticas possíveis, pela maioria dos autores consultados, como forma de minimizar o sangramento, bem como restringir a abertura do campo cirúrgico e remover uma quantidade limitada de dentes a cada sessão. ${ }^{9}$

Para uma maior segurança, tanto do paciente, como do cirurgião-dentista, o profissional pode fazer a requisição do coagulograma (Tabela 1), que é um exame de triagem utilizado para se ter acesso aos reais parâmetros hematológicos e hemostáticos do paciente. Através do coagulograma, é possível avaliar qualitativamente e quantitativamente as plaquetas, ou seja, a qualidade e a eficiência da coagulação do paciente. Este é composto por: TS - tempo de sangramento; TC - tempo de coagulação; TP ou TAP - Tempo de protrombina ativada; TTPa - Tempo de protrombina parcialmente ativada; INR - Índice de normalização internacional. ${ }^{10}$

A avaliação clínico-laboratorial dependerá fundamentalmente de uma história clínica bem detalhada e estudada, de exame clínico e físico dirigido para a procura de sinais indicativos de distúrbios hemostáticos e da solicitação precisa e interpretação correta dos exames.

Atualmente, o Índice Internacional Normalizado (INR) é o teste mais utilizado mundialmente para monitorização e planejamento pré-cirúrgico, e deve ser obtido dentro de 24 horas antes da realização dos procedimentos cirúrgicos odontológicos. ${ }^{7}$
O INR se trata do cálculo realizado entre o tempo de protrombina e a média do intervalo normal da coagulação. Após a obtenção deste valor (INR), é feita uma correção multiplicando-se pelo Índice de Sensibilidade Internacional (ISI), que tem como base umaavaliação quantitativa da resposta da tromboplastina, em comparação com o valor de referência. ${ }^{1}$

É importante destacar que cada paciente que faz uso da terapia de anticoagulante oral apresenta um grau diferente do efeito da mesma e que dependendo do tipo de doença apresentada por ele, valores maiores de INR são considerados terapêuticos. ${ }^{11,12}$

Os pacientes que não utilizam nenhum tipo de antiagregante plaquetário, anticoagulante orais ou não são hepatopatas devem possuir um valor de INR entre 0,9 a 1,0. Já os valores para os indivíduos que fazem uso de medicação, ou possuem alguma alteração no processo de coagulação, podem variam de 2 a $4 .{ }^{9,11,12}$

A OMS preconiza que é seguro realizar procedimentos cirúrgicos odontológicos com valores de INR variando entre 2 e 3,5 (Tabela 2), desde que limitados à exodontias de três elementos dentários ou instalação de três implantes de uma única vez. Na obtenção de um valor do INR acima de 5 , não se deve realizar nenhum procedimento, sendo recomendada uma consulta com o médico prescritor, para planejar a melhor forma de atendimento. ${ }^{1}$

A realização de cirurgia oral em regime ambulatorial13 poderá ser pouco segura em indivíduos com indicação de anticoagulação oral crônica e comorbidades que afetem a hemostasia ou aumentem o risco hemorrágico como: hipertensão arterial sistêmica não controlada; insuficiência renal ou hepática; alcoolismo; trombocitopenia; hemofilia ou outras perturbações da coagulação e tratamento com citostáticos. Portanto, deve-se ponderar a hospitalização do mesmo antes do procedimento cirúrgico, para rigoroso ajuste do INR e vigilância das complicações hemorrágicas.

Tabela 1. Coagulograma

\begin{tabular}{|l|l|}
\hline Exames & Valor de Referência \\
\hline Tempo de Protrombina (TP) & 11 a 15 segundos \\
\hline Tempo de tromboplastina parcialmente ativada (TTPa) & 25 a 35 segundos \\
\hline Indice normatizado internacional (INR) & 0,9 a 1,2 segundos \\
\hline
\end{tabular}

Fonte: Gerzson et al1, 2016. 
Tabela 2.Valores de referência do INR relacionado à cirurgia odontológica

\begin{tabular}{l|l|}
\hline Exames & Valor Referência \\
\hline Exodontia simples (1 elemento) & INR $\leq 4,0$ \\
\hline Exodontia de terceiros molares ou exodontias múltiplas & $\mathrm{INR} \leq 3,5$ \\
\hline Casos em que se espera grande sangramento & $\mathrm{INR}<3,0$ \\
\hline Não realizar nenhum procedimento cirúrgico & $\mathrm{INR}>5$ \\
\hline
\end{tabular}

Fonte: Gerzson et al1, 2016.

\section{Discussão}

Quando os doentes são submetidos a extração dentária sob terapêutica com anticoagulantes orais, a grande maioria das complicações hemorrágicas pós-operatórias não são secundárias à terapêutica anticoagulante oral. ${ }^{13}$ Noventa por cento dessas complicações são devidas a outras causas, incluindo traumatismo cirúrgico excessivo, má adesão do doente às instruções pós-operatórias, uso inapropriado de analgésicos como a aspirina e outros anti-inflamatórios não esteroides ou hipertensão arterial não controlada.

A fim de reduzir o risco de sangramento, estudos $^{14}$ recomendam que procedimentos múltiplos sejam divididos em várias sessões, lembrando que a complexidade do procedimento é também determinada pelo fato de envolver osteotomia e/ou retalho. A incidência de hemorragia pós-operatória foi maior após extrações dentárias que envolviam retalho e/ou osteotomia.

Em um estudo ${ }^{15}$ foi encontrado relação estatisticamente significativa entre maior valor de INR, número de dentes extraídos e risco de sangramento pós-operatório. Ainda, relataram que, dos 9 casos de sangramento pós-operatório, que correspondeu a 2,1\% da amostra, 3 casos foram relacionados a extrações simples e 6 casos a extrações múltiplas.

Em relação aos níveis de INR aceitos para cirurgias orais, de acordo com outros estudos ${ }^{16,8}$, de maneira geral, o intervalo do INR deve ficar entre 2.0 e 3.5, mas, dependendo do tipo de doença apresentada pelo paciente, valores maiores de INR são considerados terapêuticos. Nessa mesma linha de pesquisa, a maioria das diretrizes defende a realização de cirurgias dento alveolares, (exodontia de três elementos ou instalação de três implantes na mesma sessão, por exemplo) sem qualquer alteração na terapia, nos pacientes com INR inferior a $3,5 .{ }^{1}$

De acordo com as recomendações internacionais atuais os pacientes com INR superior a 4,0 apresentam um aumento considerável do risco de sangramento sem benefício clínico. ${ }^{4}$
Alguns estudos ${ }^{17,18}$ afirmam que a interrupção da terapia com anticoagulantes orais antes de procedimentos odontológicos não está embasada na literatura, uma vez que há estudos que recomendam exatamente o contrário. $\mathrm{O}$ processo causado pela suspensão da medicação expõe o paciente a um evento denominado "hipercoagulação rebote". Esse fenômeno ocorre pelo aumento na produção de protrombina e/ou ativação das plaquetas, predispondo ao tromboembolismo e podendo levar o paciente a óbito.

Corroborando com a ideia de não suspensão ${ }^{7}$, o risco do desenvolvimento de complicações tromboembólicas supera o risco de hemorragia durante procedimentos cirúrgicos orais em pacientes com intervalos de INRs terapêuticos pequenos, não existindo fortes evidencias para descontinuação da medicação uma vez que a hemorragia pós-operatória advinda do procedimento pode ser facilmente controlada através de medidas de hemostasia local.

Outros autores ${ }^{13}$ ainda relataram que o doente deve ser informado da importância de permanecer em repouso durante as primeiras duas a três horas de pós-operatório; evitar lavar a boca durante 24 horas; evitar sugar ou tossir fortemente e não tocar no local da intervenção com a língua ou com materiais estranhos. Deve ser recomendada uma dieta líquida e fria, no primeiro dia de pós-operatório. A aplicação de gelo na face por períodos de 20 minutos, de $12 \mathrm{em} 12$ horas, é útil na diminuição do risco hemorrágico e uma importante medida analgésica. O paciente deve também ser aconselhado a evitar analgésicos anti-inflamatórios não esteroides, preferindo paracetamol ou inibidores seletivos da COX-2.

\section{Conclusão}

Diante dos estudos revisados observa-se uma nova conduta no que diz respeito aos tratamentos cirúrgicos orais em pacientes submetidos á terapia anticoagulante. O planejamento compartilhado com o médico permite o cuidado adequado a esse tipo de paciente, além de permitir a tomada conjunta de decisão quanto à melhor 
conduta.

Para uma maior segurança, exames préoperatórios devem ser pedidos como: hemograma completo e coagulograma, no qual teremos acesso ao valor do INR do paciente.

A manutenção da terapia anticoagulante vem sendo priorizada, associada ao uso dos princípios de técnica cirúrgica atraumática, ao valor de INR dentro do considerado aceitável, e ao uso de medidas hemostáticas locais, para reduzir o risco de acidentes hemorrágicos. Dentre os valores aceitos de INR temos: menor ou igual a 4, para exodontia de apenas um elemento; valores entre 2 e 3,5 para cirurgias de exodontias até de 3 elementos; INR com valor igual ou de 5 impede qualquer paciente de ser submetido á procedimentos cirúrgicos.

A suspensão dos níveis terapêuticos das medicações antitrombóticas não vem sendo priorizada, devido às complicações tromboembólicas. Apesar de o índice ser baixo, às consequências desses eventos tromboembólicos são devastadores quando comparado aos eventos hemorrágicos.

\section{Referências:}

1. Gerzson AS, Lorenzo G, Lopes LAZ, Gallicchio LHH. Cirurgias odontológicas em pacientes sob terapia com antiagreganteplaquetário e anticoagulante oral: Revisão de literatura. Dental Press Publishing - J ClinDent Res. 2016 Apr/June; 13(2):98-105.

2. Kreuger MR, Diegoli MN, Pedrini RA, Porfírio VR, Silva F. Consulta Odontológica e doença sistêmica: Análise do conhecimento dos cirurgiões dentistas em Itajaí-SC. FOL, Faculdade de Odontologia de Lins/Unimep. 2009; 21(2):15-22.

3. Castro IN, Tibúrcio RC, Souki MA. Reversão de urgência da anticoagulação. RevMed Minas Gerais. 2014; 24 (Supl3): S49-S59.

4. Pesse MS, Macedo LD, Mestriner SF, Bataglion CAN. Protocolo de atendimento odontológico a pacientes usuários de terapia antitrombótica, RFO UPF - Passo Fundo. 2018 maio/ago.; 23(2): 229-235.

5. Gomes DFN. Cirurgia oral em pacientes sob terapia anticoagulante. Dissertação (Mestrado em Medicina Dentária) Instituto superior de ciências da saúde Egas Moniz. 2016; 61p.

6. Junior OC. Ponderações nos procedimentos cirúrgicos odontológicos em pacientes sob terapia antitrombótica: Revisão de literatura. Rev. bras. odontol. Rio de Janeiro. 2016 out./dez.; 73(4): 315-319.

7. Pedrosa MS, Galdino JCS, Ferro FED, Pompeu JGF, Borba MSC. Cirurgias orais em pacientes em uso de Varfarina. Revista Bahiana de Odontologia. 2016 Jun7(2):132-139

8. Araujo FM Lopes MVN, Alvarenga RL, Alvarenga GL, Souza LN. Cirurgia Oral em Pacientes sob Terapia com Anticoagulantes Orais: Revisão da Literatura e Apresentação de Dois Casos Clínicos. Volume 51, N4, 2010 .

9. Amarala COF, Nascimento FM, Pereira FD, Parizia AGS, Straiotoa FG, Amaralb MSP. Bases para Interpretação de Exames Laboratoriais na Prática Odontológica. UNOPAR CientCiêncBiol Saúde. 2014; 16(3): 22937.

10. Siqueira CR, Machado FAR Souza SMF. Terapia Anticoagulante em candidatos a cirurgia oral: Revisão de literatura, InterdisciplinaryScientificJournal. 2017 Oct-Dec; 4(5):152-162.

11. Mouchrek MMM, Frazão MCA, Costa MA Silva GQTL, Pereira EM, Cruz MCF. Exodontia em paciente usuário de anticoagulante. Faculdade de Odontologia de Lins/Unimep. 2015 jan.-jun.; 25(1) 47-54
12. Lourenço DM, Lopes LHC, Vignal CV, Morelli VM. Avaliação Clínica e Laboratorial de Pacientes em Uso de Anticoagulantes Orais. ArqBrasCardiol. 1997; 68 (5): 353-356.

13. Antônio N, Castro G, Ramos D, Machado A, Gonçalves L, Macedo T, Providência LA. Controvérsias na Anticoagulação Oral: Continuar ou Interromper os Anticoagulantes Orais durante a Intervenção Estomatológica? RevPortCardiol. 2008; 27: 531-44

14. Hanken H, Grobe A, Heiland M, Smeets R, Kluwe L, WiknerJ, et al. Postoperative bleeding risk for oral surgery under continued rivaroxaban anticoagulant therapy. Clin Oral Invest, 2016; 20(6): 1279-82.

15. Febbo A, Cheng A, Stein B, Goss A, Sambrook P. Postoperative bleeding following dental extractions in patients anticoagulated with warfarin. J Oral Maxillofac Surg. 2016 Aug; 74(8): 1518-23.

16. Dantas AK, Deboni MCZ, Piratininga JL. Cirurgias odontológicas em usuários de anticoagulantes orais. Rev. Bras. Hematol. Hemoter. 2009.

17. Ferreira JS, GIL VM. Risco Cardiovascular Associado à Interrupção dos AntiagregantesPlaquetares e Anticoagulantes Orais. RevPortCardiol. 2009; 28:845-58.

18. Dinkova A, Kirova D, Delev D. Management of pacients on anticiagulant therapy undergoing dental surgical procedures. Review Article, Journal of IMAB. 2013; 19(4): 321-326. Disponívelem: http://dx.doi. org/10.5272/jimab.2013194.321 